\title{
Relaciones genéticas basadas en marcadores ISTR entre formas silvestres, cultivadas e intermedias de frijol de guía colectado en Jalisco, México
}

\section{Genetic relationships based on ISTR markers among wild, domesticated and intermediate forms of climbing bean from Jalisco, Mexico}

\author{
Osvaldo A. Castellanos-Hernández' (10), Rogelio Lepiz-IIdefonso² (1) Guillermo Eduardo Castellanos-Enríquez² (D), \\ Araceli Rodríguez-Sahagún' (i) y Martha Isabel Torres-Morán²,3 (D)
}

1 Universidad de Guadalajara, Centro Universitario de la Ciénega, Avenida Universidad 1115, Colonia Lindavista, 47820 Ocotlán, Jalisco, México.

2 Universidad de Guadalajara, Centro Universitario de Ciencias Biológicas y Agropecuarias, km 15.5 carretera a Nogales, 45101 Zapopan, Jalisco, México.

3 Autor para la correspondencia: isabel.torres@academicos.udg.mx

Citar como:

Castellanos-Hernández, O. A., R. Lepiz-Ildefonso, G. E. Castellanos-Enríquez, A. Rodríguez-Sahagún y M. I. Torres-Morán. 2017. Relaciones genéticas basadas en marcadores ISTR entre formas silvestres, cultivadas e intermedias de frijol de guía colectado en Jalisco, México. Acta Botanica Mexicana 118: 53-63. DOI: http://dx.doi.org/10.21829/ abm118.2017.1200

Recibido: 24 de julio de 2015

Revisado: 8 de julio de 2016.

Aceptado: 19 de septiembre de 2016

DOI:

hetp://dx.doi.org/10.21829/abml18.2017.1200

\section{Resumen:}

Antecedentes y Objetivos: En el frijol de guía (Phaseolus vulgaris) se distinguen tres formas principales: cultivada, intermedia y silvestre. El concepto de forma se utiliza como categoría jerárquica dentro de una especie de acuerdo a la divergencia morfológica y genética, distribución ecogeográfica, posibilidades de hibridación y fertilidad de los híbridos y sus derivados. El objetivo fue detectar las relaciones genéticas entre poblaciones silvestres, intermedias y cultivadas provenientes de una región en el occidente de México.

Métodos: Se realizó un análisis en 30 poblaciones de frijol común de guía con datos moleculares obtenidos con el marcador ISTR (Inverse Sequence Tagged Repeat), basado en secuencias de retrotransposones copia-like. Se calculó la similitud por coeficiente de Jaccard entre cada planta analizada. Se determinó la estructura genética usando el modelo Bayesiano probabilístico.

Resultados clave: Se encontró un alto grado de diferenciación genética $\left(\mathrm{F}_{\mathrm{ST}}\right)$ entre las formas cultivadas y las silvestres. Se infirió el número de grupos asociados a la hibridación entre los materiales, basados en el coeficiente de similitud. El análisis de estructura genética detectó nueve grupos dentro del material estudiado. El marcador ISTR se presenta como un marcador efectivo para diferenciar material cultivado del silvestre y establecer grados de similitud entre las accesiones.

Conclusiones: Se detectaron asociaciones entre las variedades cultivadas y accesiones intermedias, así como una clara separación de las cultivadas de las silvestres. Esta información genera datos valiosos para la caracterización de la variabilidad genética de este recurso, así como la obtención de datos importantes para el diseño de planes de conservación y mejoramiento.

Palabras clave: conservación, frijol de guía, mejoramiento, retrotransposones, variabilidad genética.

\section{ABSTRACT:}

Background and Aims: In the climbing beans (Phaseolus vulgaris) three main forms are distinguished: cultivated, semi-wild and wild. The concept of form is used as a hierarchical category within a species according to morphological and genetic divergence, ecogeographic distribution, possibilities of hybridization and fertility of hybrids and derivatives. The objective was to investigate the genetic relationships between wild, semi-wild and cultivated bean populations from a region in western Mexico. Methods: An analysis of 30 common bean accessions with the molecular marker ISTR (Inverse Sequence Tagged Repeat) was realized, based on sequences of retrotransposons copia-like. The similarity among individual plants was calculated with Jaccard's coefficient. Genetic structure was determined using a probabilistic Bayesian model.

Key results: A high level of genetic differentiation $\left(\mathrm{F}_{\mathrm{ST}}\right)$ was determined between cultivated and wild beans. The number of groups associated with hybridization was inferred, based on the similarity coefficient method. Analysis of genetic structure detected nine groups within the material studied. The ISTR marker is presented as an effective marker to distinguish between cultivated and wild material and establish the degree of similarity between accessions.

Conclusions: Cultivated beans were associated with semi-wild accessions and clearly separated from wild ones. This information generates valuable data for the characterization of the genetic variability of this resource, as well as for assessing germplasm conditions for conservation and breeding programs.

Key words: breeding, climbing bean, conservation, genetic variability, retrotransposons. 


\section{INTRODUCCIÓN}

El conocimiento de las características genéticas de un recurso vegetal representa un importante prerrequisito para su conservación y uso. El frijol (Phaseolus vulgaris L.) es un cultivo que en la actualidad ha cobrado mucha importancia en países alejados de su lugar de origen y distribución como Etiopía, Slovenia y Uganda (Gebeyehu et al., 2006; Mwale et al., 2009; Worku, 2013). En México siempre ha formado parte de la alimentación básica. En Jalisco se realizaba tradicionalmente la siembra de frijol de guía en asociación con maíz, sobre todo en algunas zonas de cultivo de temporal. Esto ha sido modificado por la necesidad de incrementar los rendimientos y por el desarrollo de tecnología propia para este cultivo ahora manejado en espaldera (Gebeyehu et al., 2006; Lépiz et al., 2015).

Las poblaciones utilizadas en el presente estudio difieren principalmente en el grado de manejo al que fueron o están siendo sujetas por el hombre, lo cual es notable también en los caracteres morfológicos. En el frijol de guía se distinguen tres formas principales: cultivada, intermedia y silvestre. El concepto de forma se utiliza como categoría jerárquica dentro de una especie de acuerdo a la divergencia morfológica y genética, distribución ecogeográfica, posibilidades de hibridación, y fertilidad de los híbridos y sus derivados (Lépiz, 2014). Las formas se distinguen de manera general con base en una sola característica morfológica, es decir, su hábito trepador, sus rangos geográficos son simpátricos, la divergencia genética está bajo control genético simple, la hibridación es común y los híbridos son completamente fértiles, además de la notable diferencia en el tamaño y forma de las semillas (Lépiz et al., 2010; Poczai et al., 2013; Lépiz, 2014). La variabilidad genética del frijol se encuentra expuesta a diversas presiones que fomentan la uniformidad de las variedades comerciales y reducen la variación genética de las distintas formas de la especie (cultivada, intermedia y silvestre). Bitocchi et al. (2012) reportaron la diversidad de frijol existente hasta el presente siglo y confirmaron el origen mesoamericano de esta especie así como la diversidad mayor en las líneas originarias de esta región contra la presente en los frijoles andinos. Esos estudios estuvieron basados en la comparación de frijoles silvestres de cada origen (Mesoamericano y Andino) contra los cultivados encontrados en ambas regiones y ubicaron al Occidente de México entre los sitios con mayor diversidad en esta especie.

Algunas poblaciones de frijol silvestre han desaparecido por completo y en otras se ha reducido significativamente el número de individuos con la consecuente deriva genética (Rosales-Serna, 2009; Lépiz, 2014). Además, en México la forma domesticada de esta leguminosa se enfrenta a modificaciones importantes ante una sociedad cambiante en hábitos alimenticios. Al respecto, es importante mencionar que los estudios de caracterización que incluyen líneas o colectas de especies silvestres tienen la ventaja de proporcionar información de genes que no están incluidos en el germoplasma cultivado y que pueden ser usados como fuente de mejoramiento genético (Burle et al., 2010; Bitocchi et al., 2012; Hernández-López et al., 2013; Govindaraj et al., 2015).

La importancia de este estudio radica en la posibilidad de caracterizar y diferenciar, a través del uso de herramientas moleculares, las diferentes formas de frijol común de guía (silvestres, intermedias y cultivadas) pertenecientes al complejo genético mesoamericano (Blair et al., 2009). Se trata de colectas realizadas en una zona que fue muy importante para el cultivo de frijol asociado con maíz y donde se encontraban frijoles silvestres que posteriormente produjeron las líneas intermedias. Esta asociación maíz-frijol actualmente no se utiliza y el cultivo de frijol de guía tiende a ser manejado en espalderas. Este trabajo tiene la finalidad de definir sus diferencias y similitudes genéticas generando información valiosa para la caracterización de la variabilidad de este recurso fitogenético, así como en la obtención de datos importantes para el diseño de planes de conservación y mejoramiento.

Para ello, se utilizó el marcador molecular ISTR (Inverse Sequence Tagged Repeat) que está basado en secuencias de retrotransposones copia-like, un mecanismo de transposición similar a la familia de virus Metaviridae, cuya presencia se ha reportado para esta especie en 
un número de 40 secuencias (Galindo et al., 2004; Xiao et al., 2004). Los retrotransposones contienen secuencias muy conservadas LTR (Long Terminal Repeat) que contienen genes que codifican para proteínas estructurales y enzimas, que se utilizan para la síntesis de los iniciadores de marcadores moleculares como los ISTR (Pearce et al., 1996; Kalendar et al., 2011; Dongying et al., 2016). Los marcadores de este tipo han sido utilizados en muchos estudios de variabilidad y diversidad genética ya que se ha probado que las mutaciones inducidas por este tipo de transposición son usualmente muy estables en contraposición con los transposones, y el polimorfismo que detectan ha permitido estudios de etiquetado de genes y diversos análisis funcionales (Aga y Bryngelsson, 2006; Zou et al., 2009; Jing et al., 2010).

\section{Materiales y Métodos}

\section{Material vegetal}

El material vegetal para este estudio consistió en semillas de 30 colectas de frijol de guía de las cuales cinco corresponden a formas cultivadas o domesticadas, cinco silves- tres y 20 intermedias (Cuadro 1). Todas fueron colectadas en el estado de Jalisco con la excepción de una que proviene de Nayarit (Fig. 1). La variación de tamaño y color de las semillas se muestra en la figura 2. Las colectas permanecen conservadas como accesiones del Banco de Germoplasma del Centro Universitario de Ciencias Biológicas y Agropecuarias de la Universidad de Guadalajara (CUCBA). Se entiende como accesión un volumen de semilla asignado, con número secuencial y de identificación de colecta, al banco de germoplasma y a una base de datos nacional o internacional (Aubry et al., 2005).

Las semillas de cada población fueron sembradas en invernadero en el CUCBA y cultivadas hasta su floración. De cada población se tomó al azar tejido foliar de 10 individuos obteniendo un total de 300 individuos para el estudio.

\section{Análisis molecular}

Se obtuvo el ADN de los individuos de cada colecta usando el método reportado por Saghai-Maroof et al. (1984), se cuantificó y evaluó por espectrofotometría y por electroforesis en geles de agarosa a 1\% (Sambrook y Russell,

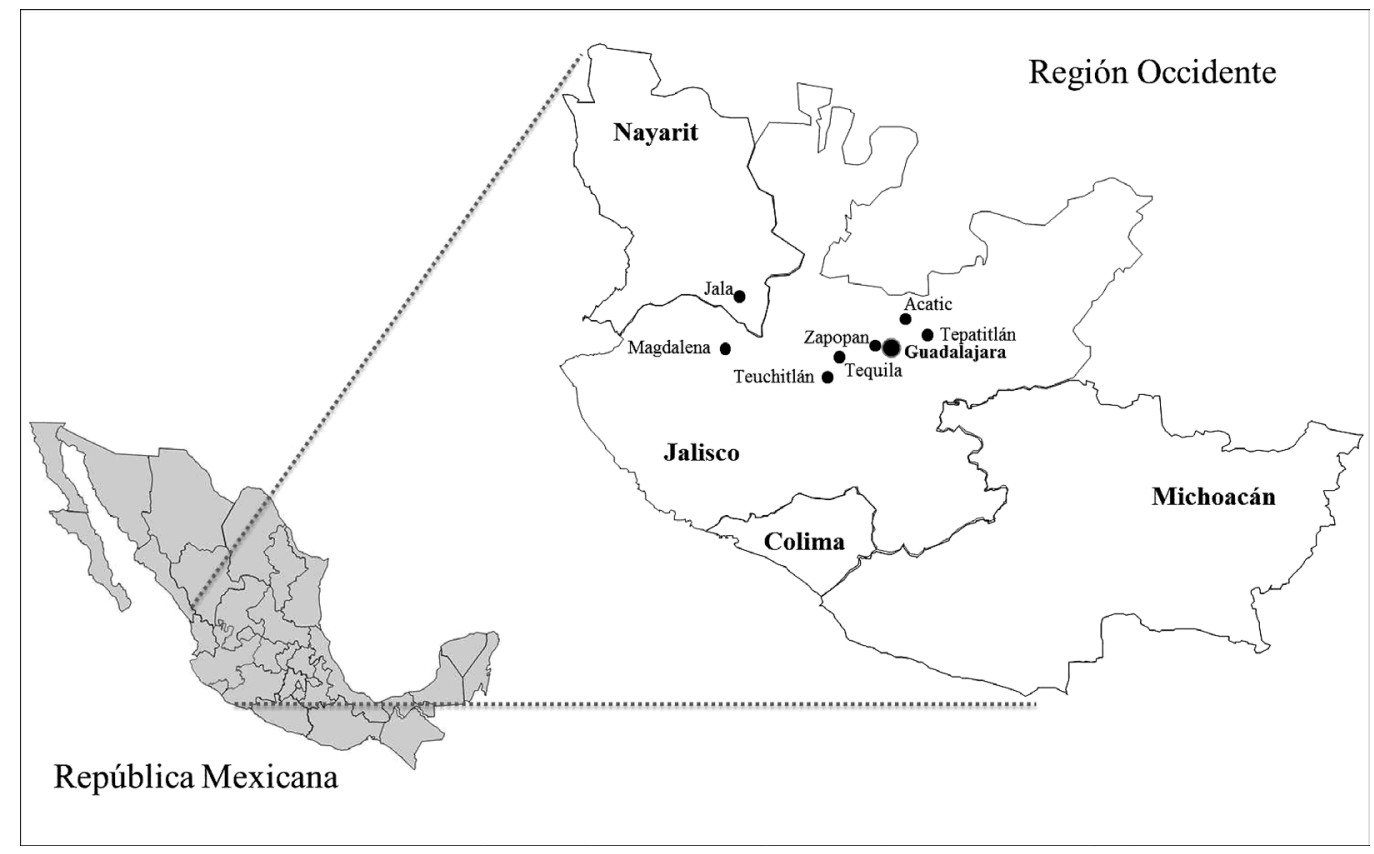

Figura 1: Ubicación de los sitios de colecta para las accesiones de frijol silvestre e intermedio. 
Cuadro 1: Características de las poblaciones utilizadas en el presente trabajo. $\mathrm{C}=$ cultivada, $\mathrm{I}=$ intermedia y $\mathrm{S}=$ silvestre. $*$ Municipio de Etzatlán, Jal.

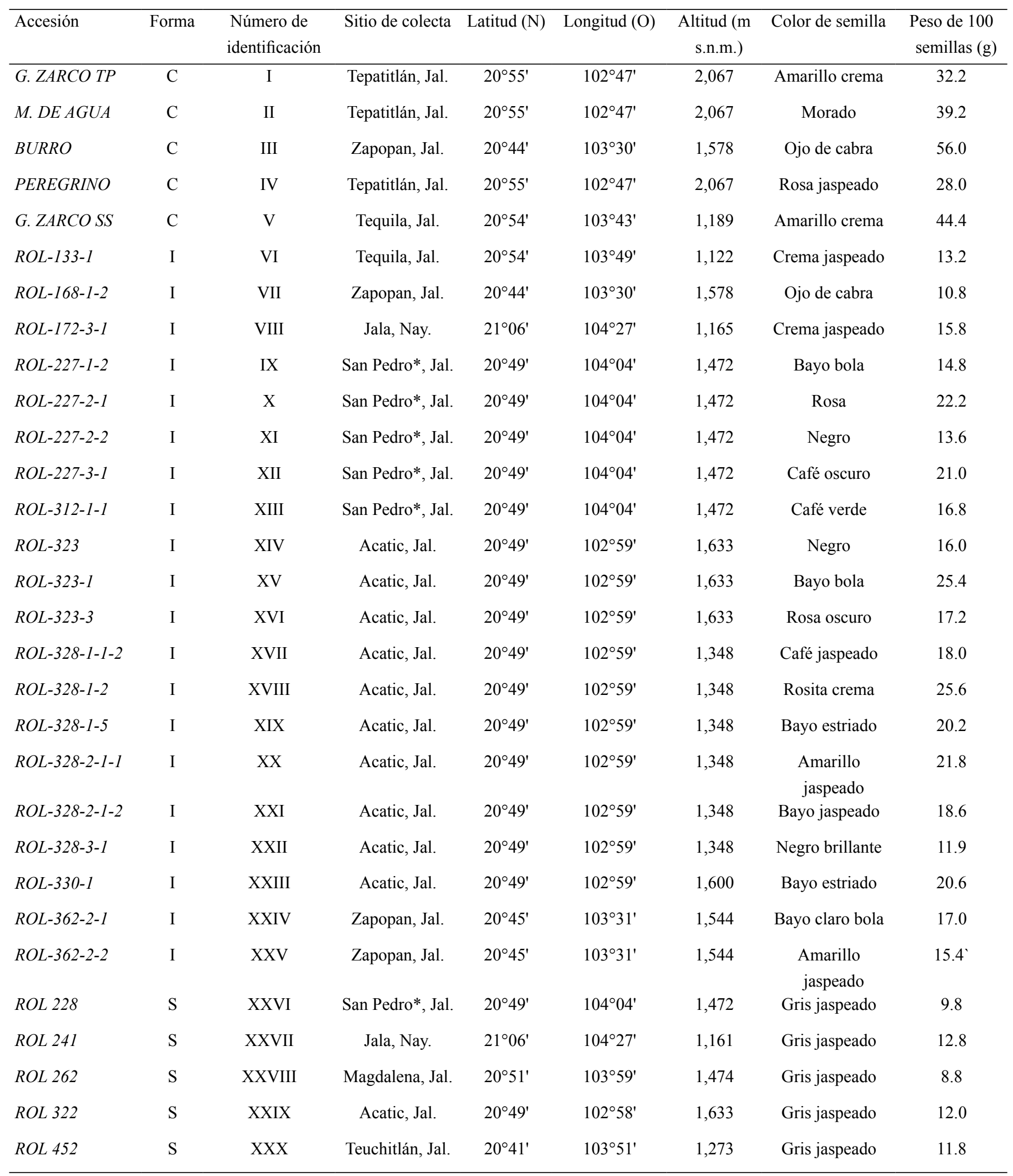




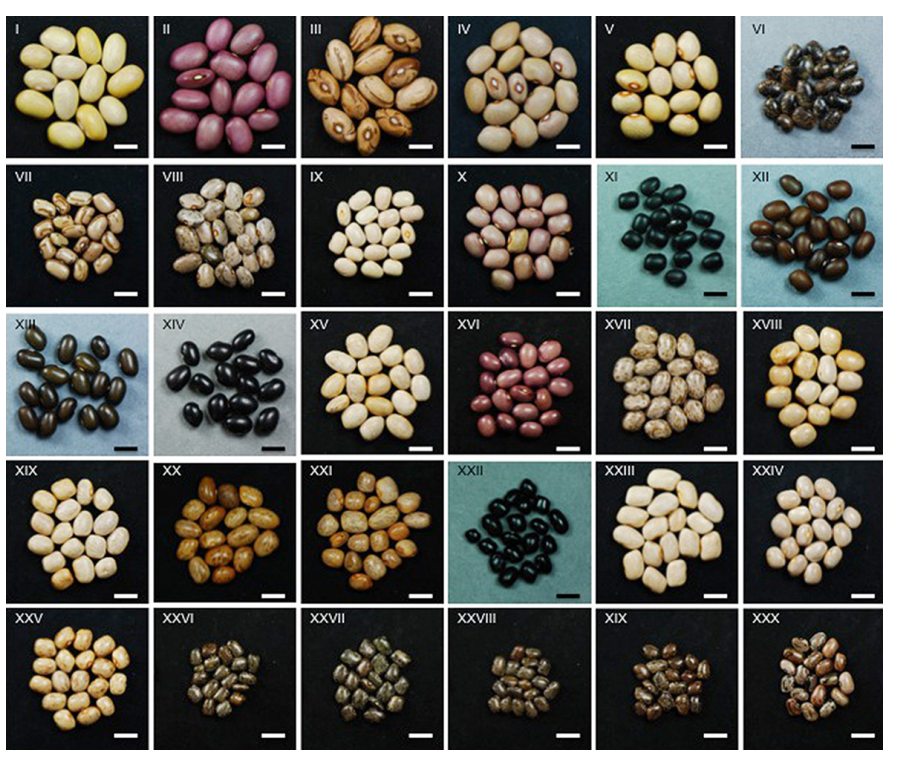

Figura 2: Semillas de frijol utilizadas en el presente estudio. I-V. cultivadas; VI-XXV. intermedias; XVI-XXX. silvestres. Barra $=0.5 \mathrm{~cm}$.

2001). Para la amplificación se utilizó el protocolo reportado por Torres-Morán et al. (2008) para ISTR, en un volumen final de $20 \mu \mathrm{L}$ utilizando $25 \mathrm{ng}$ de ADN, amortiguador PCR $1 \mathrm{X}$ (pH 7.2 Tris-HCl), $3 \mathrm{mM}$ de $\mathrm{MgCl}_{2}$, $0.3 \mu \mathrm{M}$ de cada iniciador, $0.25 \mathrm{mM}$ de dNTPs y Taq polimerasa 1U. Las condiciones de amplificación fueron las siguientes: un primer ciclo de desnaturalización de $95{ }^{\circ} \mathrm{C}$ por 3 min, seguido de 40 ciclos de desnaturalización a 95 ${ }^{\circ} \mathrm{C}$ por $3 \mathrm{~s}$; alienación a $45^{\circ} \mathrm{C}$ por 1 min y extensión a 72 ${ }^{\circ} \mathrm{C}$ por $2 \mathrm{~min}$, con una extensión final de $10 \mathrm{~min}$ a $72{ }^{\circ} \mathrm{C}$ y una temperatura de almacenamiento a $4{ }^{\circ} \mathrm{C}$. Se emplearon dos combinaciones de iniciadores F91/B31 y F10/B11, en donde $\mathrm{F}$ indica iniciador Forward (hacia adelante) y $\mathrm{B}$ Backward (hacia atrás). Las secuencias de cada uno de los iniciadores se indican en el cuadro 2.

\section{Análisis de los datos}

A partir de matrices binarias de presencia ausencia, se calculó el coeficiente de similitud de Jaccard de acuerdo a lo reportado por Lowe et al. (2004). Posteriormente se realizó un procedimiento Bootstrap o remuestreo con 10,000 iteraciones y análisis de agrupamiento por el mé-
Cuadro 2: Secuencias de los iniciadores utilizados en el presente trabajo, tomadas de Aga y Bryngelsson (2006).

\begin{tabular}{cc}
\hline INICIADOR & SECUENCIA 5 ' -3, \\
\hline F91 & ATA TGG ACT TAA GCA AGC CA \\
B31 & ATT CCC ATC TGC ACC AAT \\
F10 & TAA GCA AGC ATC TCG GAG \\
B11 & ATC AGG AAG GTC TGT AAA GC
\end{tabular}

todo UPGMA (Unweighted Pair Group with Arithmetic Average), utilizando el programa estadístico NTSYS v. 2.11 (Rohlf, 2002). Finalmente, se analizó la estructura genética de las poblaciones por medio del programa STRUCTURE (Pritchard et al., 2000), basado en el modelo Bayesiano de probabilidad condicional, para lo cual se usaron 5000 iteraciones por 5000 repeticiones. Los resultados se presentan en forma gráfica.

\section{Resultados}

Se produjeron un total de 660 bandas (loci); el porcentaje de polimorfismo detectado fue diferente para cada combinación de iniciadores. Los valores se reportan en el cuadro 3.

El análisis de agrupamiento mostró una separación de las poblaciones en ocho grupos, lo cual corresponde a una clara división entre las formas silvestres y cultivadas, ubicando poblaciones intermedias asociadas a las formas anteriores que cuentan con secuencias que coinciden o bien con las cultivadas o con las silvestres. Tal es el caso

Cuadro 3: Número de bandas polimórficas y porcentaje de polimorfismo detectado con dos combinaciones de iniciadores de ISTR en las poblaciones de Phaseolus vulgaris L.

\begin{tabular}{lccc}
\hline Iniciadores & $\begin{array}{c}\text { Total de bandas } \\
\text { producidas }\end{array}$ & $\begin{array}{c}\text { Número de bandas } \\
\text { polimórficas }\end{array}$ & $\begin{array}{c}\text { Porcentaje de } \\
\text { polimorfismo }\end{array}$ \\
\hline F91/F31 & 480 & 427 & 88.95 \\
F10/B11 & 180 & 153 & 85.00 \\
TOTAL & 660 & 580 & 87.87 \\
\hline
\end{tabular}


de las poblaciones VI, VII, VIII, IX, X y XI que fueron agrupadas con las cultivadas y las XXII y XXV que fueron ubicadas con las silvestres. Las XII, XIII, XIV, XV, XVI, XVII, XVIII, XIX, XX, XXI, XXII y XXIII se agruparon de manera independiente, todas correspondientes al grupo de frijoles con forma intermedia.

El coeficiente de Jaccard mostró un grado de similitud máximo de 0.66 en el cual se ubicaron las
XVI y XVII, por lo que se puede concluir que son las dos poblaciones más parecidas genéticamente con base en secuencias detectadas por ISTR. El grado de similitud más bajo con base en el coeficiente de Jaccard fue de 0.17, en donde se ubican las II y XXX, siendo éstas las menos parecidas genéticamente, ya que corresponden a una población de frijol cultivado y una silvestre (Fig. 3).

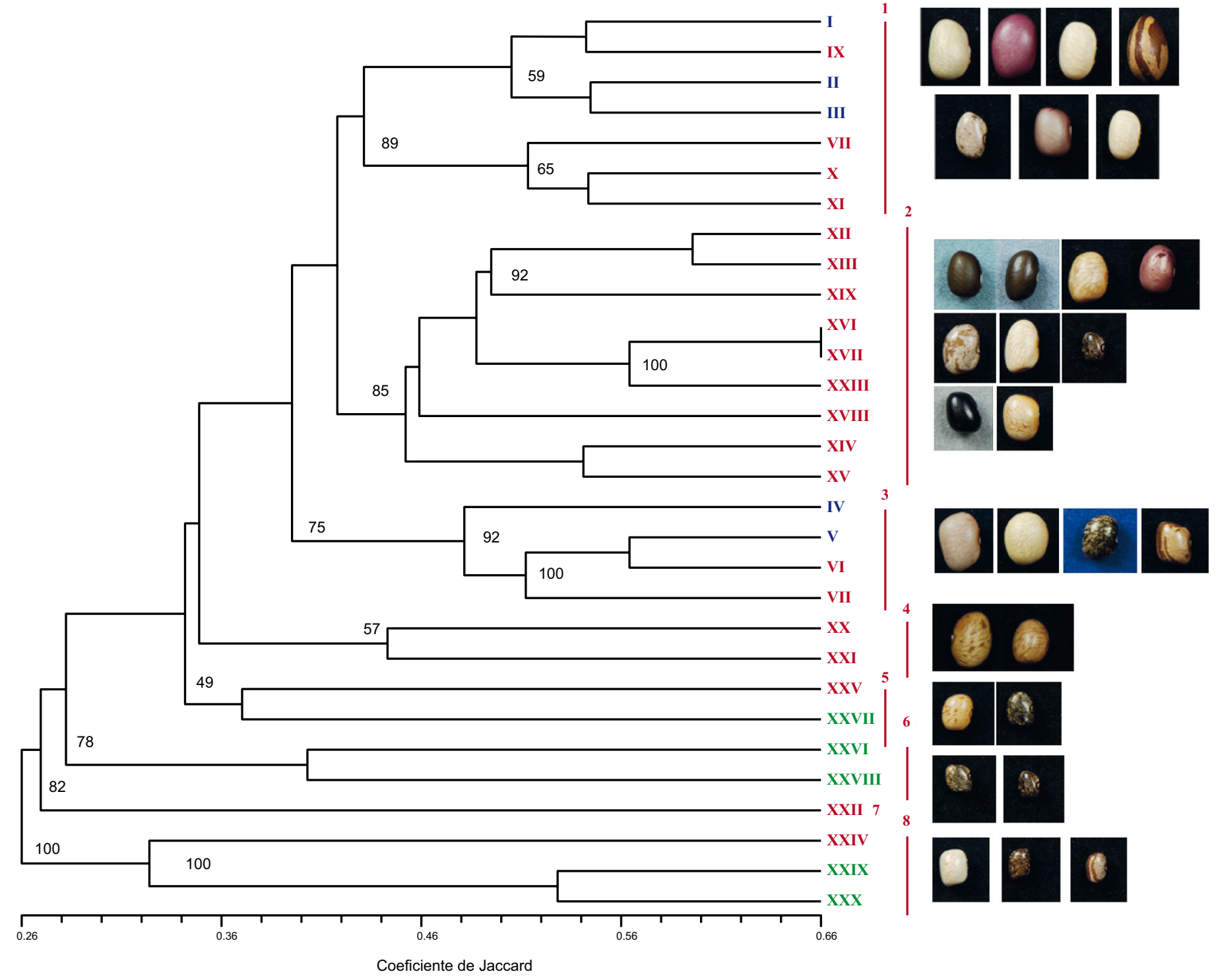

Figura 3: Dendrograma obtenido con remuestreo (Bootstrap) y análisis de agrupamiento a partir de las matrices generadas con ISTR. Se muestra la asociación en ocho grupos de las accesiones analizadas y los valores de confiabilidad del remuestreo. Poblaciones marcadas con azul = cultivadas, rojo $=$ intermedias, verde $=$ silvestres. 
Los valores del remuestreo (Bootstrap) para el dendrograma obtenido abarcaron de $49 \%$ a $100 \%$ y se produjeron 8 grupos (Fig. 3). El valor máximo de similitud se encontró entre las accesiones XVI y XVII a un nivel de 0.66 del coeficiente de Jaccard, estas accesiones son provenientes de Acatic, Jalisco. Las accesiones provenientes de esta localidad fueron agrupadas principalmente en el grupo 2 .

Utilizando el programa STRUCTURE se obtuvo la diferenciación dentro de las poblaciones, donde se confirma la estructura genética del material conformada en nueve grupos diferentes, $(K=9)$, lo que difiere muy poco con lo encontrado por similitud y agrupamiento (UPGMA) en el que se encontraron ocho grupos. Los valores de diferenciación genética calculados con este programa se reportan en el cuadro 4. Los valores de diferenciación genética van de 0 a 1 donde $0=$ no diferenciación, $<0.05=$ diferenciación pequeña, $0.05<\mathrm{y}<0.15=$ diferenciación moderada, $0.15<\mathrm{y}<0.25=$ diferenciación grande, $>0.25=$ diferenciación muy grande, 1=fijación de alelos alternativos en diferentes poblaciones o subpoblaciones. Los valores indican que el mayor grado de diferenciación corresponde al grupo $1(0.7278)$ y al $6(0.6436)$, lo cual corresponde al grupo de material cultivado y a los intermedios respectivamente.

Cuadro 4: Valores de diferenciación genética $\left(\mathrm{F}_{\mathrm{ST}}\right)$ obtenidos para los nueve grupos sugeridos por el programa STRUCTURE.

\begin{tabular}{cc}
\hline Grupo & $\mathrm{F}_{\mathrm{ST}}$ (diferenciación genética) \\
\hline 1 & 0.7278 \\
2 & 0.3754 \\
3 & 0.3596 \\
4 & 0.6883 \\
5 & 0.4371 \\
6 & 0.6436 \\
7 & 0.2364 \\
8 & 0.6076 \\
9 & 0.4537 \\
\hline
\end{tabular}

En la figura 4 se muestra el resultado de cada simulación para diferente número de grupos $(K=2$ hasta $K=10$ ). Se presentan los grupos en diferente color, lo cual hace posible apreciar cómo algunos individuos de la forma intermedia comparten secuencias similares con silvestres y con cultivadas. Lo cual corresponde también al resultado obtenido por similitud. La correspondencia de colores en los individuos de las colectas de frijol intermedio que son similares a los silvestres o a los domesticados se complementa con la referencia de probabilidad de pertenencia de cada individuo a cada grupo simulado.

\section{DISCUSIÓN}

Con base en los valores del remuestreo, se puede considerar que el dendrograma obtenido es confiable y las asociaciones que se muestran se deben a la similitud genética entre las accesiones asociadas en cada grupo. La relación que se presenta en el dendrograma se asocia con el fenotipo, forma, tamaño y color de la semilla. Las plantas dentro del grupo intermedio producen po-

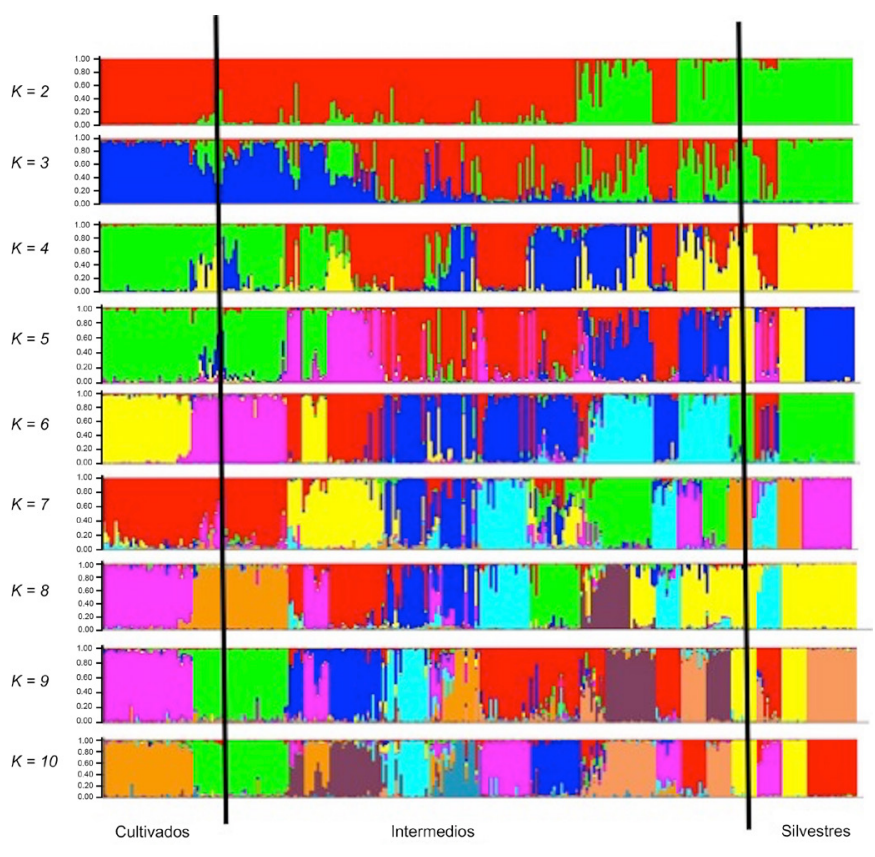

Figura 4: Análisis de estructura genética de las poblaciones, donde se muestran nueve simulaciones $(K=2$ a $K=10)$ hechas con el programa STRUCTURE. Cada simulación contiene su referencia de probabilidad de pertenencia a cada grupo simulado. 
cas semillas por vaina pero más grandes que la forma silvestre (Graham y Ranalli, 1997; Zizumbo-Villareal et al., 2005). La variación en el color de la semilla es muy alta, lo cual ha sido reportado también por Blair et al. (2009). Este grupo con forma intermedia se ha encontrado en sitios donde crecen las poblaciones silvestres, pero cercanos a parcelas donde se cultiva o se cultivó frijol asociado con maíz. Las accesiones provenientes de la localidad de Acatic, Jalisco, se ubicaron en el grupo con mayor similitud genética con niveles de 0.35 hasta 0.66 del coeficiente de Jaccard; sin embargo, se muestra una gran variabilidad en el fenotipo de semillas (Fig. 3).

Las poblaciones silvestres no han sido sometidas a ningún manejo por parte del hombre y se colectaron en hábitats libres de perturbación humana, presentan semillas pequeñas y la variación en el color de la semilla es uniforme (Lépiz et al., 2010). Estos grupos se separaron más claramente en los análisis realizados.

El porcentaje de bandas polimórficas que se obtuvieron con el marcador ISTR indica que puede ser un marcador confiable para su utilización como método para diferenciar genéticamente a las poblaciones con diferente forma de frijol común. Los frijoles intermedios forman un grupo molecularmente cercano tanto a poblaciones silvestres como cultivadas; sin embargo, algunas poblaciones se agruparon aparte, sugiriendo que forman un grupo distinto y que deben considerarse de importancia para su estudio y conservación, así como materia prima para la hibridación en busca de mejoramiento genético. Esta información puede marcar una nueva dirección en el estudio de las diferencias entre accesiones, cultivares o líneas de frijol, así como la diferenciación entre formas (Poczai et al., 2013).

La manera en que se formaron los grupos en el dendrograma demostró que este marcador detecta las variaciones en el genoma de las plantas estudiadas. Dichas variaciones son representadas por elementos transponibles y presumiblemente reflejan hibridación entre las formas cultivadas y silvestres como origen de las formas intermedias.
Los altos valores de diferenciación genética $\left(\mathrm{F}_{\mathrm{ST}}\right)$ obtenidos a partir del análisis con el programa STRUCTURE sugieren una gran diferenciación en términos de frecuencias alélicas, particularmente en las formas cultivadas e intermedias. Estos resultados sugieren probablemente mayor calidad homocigota en estas accesiones, bajo nivel de flujo genético y diferencias atribuibles a su origen geográfico (Rousset, 1997; Poczai et al., 2013).

La estructura obtenida mediante el análisis Bayesiano con STRUCTURE se considera robusta si se obtuvo de múltiples corridas independientes en el programa, como mencionan Jing et al. (2010), quienes trabajaron con especies de chícharo (Pisum L. spp.) como modelo, usando marcadores basados en retrotransposones. La agrupación de las formas intermedias con las cultivadas o las silvestres podría sugerir el origen de dichas formas como resultado de la hibridación natural entre poblaciones cultivadas y silvestres. Esto concuerda con los resultados obtenidos por Papa y Gepts (2003) a partir de AFLPs en poblaciones domesticadas, silvestres e intermedias de frijol común provenientes del complejo mesoamericano, en donde la ausencia de frijoles domesticados en la agrupación de los silvestres indica asimetría en el flujo genético entre estas dos formas, mientras que señalan el origen de los frijoles intermedios como resultado de hibridación entre los domesticados y silvestres.

Al analizar líneas de frijol similares provenientes del occidente de México con parámetros morfoagronómicos, comparando poblaciones de frijol cultivadas, silvestres e intermedias de frijol, Lépiz et al. (2010) encontraron a las poblaciones intermedias más cercanas a las silvestres, lo cual concuerda con los resultados obtenidos en el presente estudio. La conservación de las poblaciones bajo estudio es importante para disponer de la variabilidad genética existente. Se observó un mayor número de bandas obtenidas con el marcador ISTR en las poblaciones cultivadas e intermedias en comparación a las silvestres, lo que pudiera sugerir el efecto a través del tiempo en la generación de variación genética por el manejo del hombre sobre Phaseolus vulgaris. Sin embargo, esto también se puede deber a las regiones que detecta el marcador 
molecular utilizado, ya que está basado en secuencias de retrotransposones que son elementos móviles en el genoma del organismo en cuestión.

\section{CONTRIBUCIONES DE AUTORES}

MT y RL concibieron y diseñaron el estudio. MT y OC realizaron los análisis. GC y AR contribuyeron a la adquisición de los datos moleculares, trabajo de laboratorio y interpretación de los resultados. MT, OC y AR escribieron el manuscrito con la ayuda de RL. Todos los autores contribuyeron a la discusión, revisión y aprobación del manuscrito final.

\section{FINANCIAMIENTO}

Este estudio fue apoyado a través del proyecto BEI-FRI-09 auspiciado con fondos federales del Sistema Nacional de Recursos Fitogenéticos de la Secretaría de Agricultura Ganadería Desarrollo Rural, Pesca y Alimentación.

\section{LITERATURA CITADA}

Aga, E. y T. Bryngelsson. 2006. Inverse sequence-tagged repeat (ISTR) analysis of genetic variability in forest coffee (Coffea arabica L.) from Ethiopia. Genetic Resources and Crop Evolution 53: 721-728. DOI: http://dx.doi. org/10.1007/s10722-004-5729-5

Aubry, C., R. School y V. Erickson. 2005. Grass cultivars: their origins, development and use on national forest and grasslands in the Pacific Northwest. USDA Forest Service. 44 pp. www.nativeseednetwork.org/article view?id=13.

Blair, M. W., L. M. Díaz, H. F. Buendía y M. C. Duque. 2009. Genetic diversity, seed associations and population structure of core collection of common beans (Phaseolus vulgaris L.). Theoretical and Applied Genetics 119(6): 955-972. DOI: http://dx.doi.org/10.1007/ s00122-009-1064-8

Bitocchi, E., L. Nanni, E. Bellucci, M. Rossi, A. Giardini, P. Spagnoletti-Zeuli, G. Logozzo, J. Stougaard, P. McClean, G. Attene y R. Papa. 2012. Mesoamerican origin of the common bean (Phaseolus vulgaris L.) is revealed by sequence data. Proceeding of the National Academ- ic of Sciences 109(14): E788-E796. DOI: http://dx.doi. org/10.1073/pnas.1108973109

Burle, M. L., J. R. Fonseca, J. A. Kami y P. Gepts. 2010. Microsatellite diversity and genetic structure among common bean (Phaseolus vulgaris L.) landraces in Brazil, a secondary center of diversity. Theoretical and Applied Genetics 121: 801-813. DOI: http://dx.doi.org/10.1007/ s00122-010-1350-5

Dongying, G., Y. Li, K. D. Kim, B. Abernathy y S. A. Jackson. 2016. Landscape and evolutionary dynamics of terminal repeat retrotransposons in miniature in plant genomes. Genome Biology 17(7): 1-17. DOI: http://dx.doi. org/10.1186/s13059-015-0867-y

Galindo, L. M., E. Gaitán-Solís, P. Baccam y J. Tohme. 2004. Isolation and characterization of RNase LTR sequences of Ty1-copia retrotransposons in common bean (Phaseolus vulgaris L.). Genome 47: 84-95. DOI: http://dx.doi. org $/ 10.1139 / \mathrm{g} 03-102$

Govindaraj, M., M. Vetriventhan y M. Srinivasan. 2015. Importance of Genetic Diversity Assessment in Crop Plants and Its Recent Advances: An Overview of Its Analytical Perspectives. Genetics Research International 431487: 1-14. DOI: http://dx.doi.org/10.1155/2015/431487

Gebeyehu, S., B. Simane y R. Kirkby. 2006. Genotype X cropping system interaction on climbing beans (Phaseolus vulgaris L.) grown as sole crop and in association with maize (Zea mays L.). European Journal of Agronomy 24(4): 396-403. DOI: http://dx.doi.org/10.1016/j. eja.2006.01.005

Graham, P. H. y P. Ranalli. 1997. Common bean (Phaseolus vulgaris L.). Field Crops Research 53: 131-146. DOI: http://dx.doi.org/10.1016/S0378-4290(97)00112-3

Hernández-López, V. M., Ma. L. P. Vargas-Vázquez, J. S. Muruaga-Martínez, S. Hernández-Delgado y N. MayekPérez. 2013. Origen, domesticación y diversificación del frijol común. Avances y perspectivas. Revista Fitotecnia Mexicana 36 (2): 95-104.

Jing, R., A. Vershinin, J. Grzebyta, P. Shaw, P. Smýkal, D. Marshall, M. J. Ambrose, T. H. Ellis y A. J. Flavell. 2010. The genetic diversity and evolution of field pea (Pisum) studied by high throughput retrotransposon based in- 
sertion polymorphism (RBIP) marker analysis. BMC Evolutionary Biology (10)44: 1-20. DOI: http://dx.doi. org/10.1186/1471-2148-10-44

Kalendar, R., A. J. Flavell, T. H. N. Ellis, T. Sjakste, C. Moisy y A. H. Schulman. 2011. Analysis of plant diversity with retrotransposon-based molecular markers. Heredity 106: 520-530. DOI: http://dx.doi.org/10.1038/hdy.2010.93

Lépiz, R., J. López, J. Sánchez, F. Santacruz-Ruvalcaba, R. Nuño y E. Rodríguez. 2010. Características morfológicas de formas cultivadas, silvestres e intermedias de frijol común de hábito trepador. Fitotecnia Mexicana 33(1): 21-28.

Lépiz, R. 2014. Variabilidad genética y mejoramiento del cultivo de frijol. In: Solís-Gadea, H. y K. Planter-Pérez (coord.). Jalisco en el Mundo Contemporáneo Tomo IV: Ciencias Agropecuarias y Medio Ambiente. Universidad de Guadalajara-Consejo Estatal de Ciencia y Tecnología. Guadalajara, México. Pp. 105-115.

Lépiz, R., S. Sánchez, E. López, J. J. López, I. E. Chavarín y K. Meza. 2015. El cultivo de frijol en Jalisco: tecnología para altos rendimientos. Universidad de Guadalajara. Guadalajara, México. 54 pp.

Lowe, A., S. Harris y P. Ashton. 2004. Ecological genetics: design analysis and application. Blackwell Publishing. Oxford, UK. e-Book: http://samples.sainsburysebooks. co.uk/9781444311211_sample_388310.pdf

Mwale, V. M., J. M. Bokosi, C. M. Masangano, M. B. Kwapata, V. H. Kabambe y C. Miles. 2009. Performance of climber common bean (Phaseolus vulgaris L.) lines under researcher designed farmer managed (RDFM) system in three bean agro-ecological zones of Malawi. African Journal of Biotechnology 8 (11): 2460-2468.

Papa, R. y P. Gepts. 2003. Asymmetry of gene flow and differential geographical structure of molecular diversity in wild and domesticated common bean (Phaseolus vulgaris L.) from Mesoamerica. Theoretical and Applied Genetics 106: 239250. DOI: http://dx.doi.org/10.1007/s00122-002-1085-z

Pearce, S. R., G. Harrison, D. Li, J. S. Heslop-Harrison, A. Kumar y A. J. Flavell. 1996. The Ty1-copia group retrotransposons in Vicia species: copy number, sequence heterogeneity and chromosomal localization. Molecular and General Genetics 250: 305-315.
Poczai, P., I. Varga, M. Laos, A. Cseh, N. Bell, J. Valkonen y J. Hyvönen. 2013. Advances in plant gene-targeted and functional markers: a review. Plant Methods 9(6): 2-31. DOI: http://dx.doi.org/10.1186/1746-4811-9-6

Pritchard, J. K., M. Stehens y P. Donnelly. 2000. Inference of population structure using multilocus genotype data. Genetics 155: 945-959.

Rousset, F. 1997. Genetic differentiation and estimation of gene flow from F-statistics under isolation by distance. Genetics 145(4): 1219-1228.

Rohlf, F. J. 2002. NTSYSpc: Numeral taxonomy and multivariate analysis system. Ver. 2.1. Exeter Software. Setauket, USA. 42 pp.

Rosales-Serna, R. 2009. Diversidad genética del frijol. Biodiversitas 89: 7-11.

Saghai-Maroof, M., K. Soliman, A. Jorgensen y R. Allard. 1984. Ribosomal DNA spacer length polymorphisms in barley: mendelian inheritance, chromosomal location and population dynamics. Proceedings of the Natural Academy of Sciences 81(24): 8014-8018.

Sambrook, J. y D. W. Russell. 2001. Molecular cloning: A laboratory manual. Vol. 3, 3th ed. Cold Spring Harbor Laboratory Press. Nueva York, USA. 999 pp.

Torres-Morán, M. I., N. Almaraz-Abarca, A. P. Velasco-Ramírez, V. Hernández-Vargas, G. Orea-Lara, A. Cifuentes-Díaz y C. Oliver-Salvador. 2008. Taxonomic significance of ISTR to discriminate species in Agavaceae. American Journal of Agricultural and Biological Sciences 3(4): 661-665. DOI: http://dx.doi.org/10.3844/ ajabssp.208.661.665

Worku, W. 2013. Sequential intercropping of common bean and mung bean with maize in southern Ethiopia. Experimental Agriculture 50(1): 90-108. DOI: http://dx.doi. org/10.1017/S0014479713000434

Xiao, W., W. Sakamoto y Sodmergen. 2004. Isolation and Characterization of Ty1/Copia-like reverse transcriptase sequences from mung bean. Acta Botanica Sinica 46(5): 582-587.

Zizumbo-Villareal, D., P. Colunga, E. Payró, P. Delgado-Valerio y P. Gepts. 2005. Population structure and evolutions dynamics of wild - weedy domesticated complexes of 
common bean in a Mesoamerican region. Crop Science 45: 1073-1083. DOI: http://dx.doi.org/10.2135/cropsci2004.0340

Zou, J., H. Gong, T. Yang y J. Meng. 2009. Retrotransposons - a Major Driving Force in Plant Genome Evolution and a Useful Tool for Genome Analysis. Journal of Crop Science and Biotechnology 12(1): 1-8. DOI: http://dx.doi. org/10.1007/s12892-009-0070-3 